\title{
Chiara Renda
}

\section{Di aetas in aetas: considerazioni sulla storiografia di Seneca Padre e Floro}

\begin{abstract}
The partition of the history of Rome by aetates is a feature shared by both the historical work of Seneca the Elder and Florus' historiography. By providing fresh comparison between the texts of these two authors, this study corroborates Canfora's thesis that Seneca the Elder set the beginning of civil wars in 133 BCE too. As concerns their evaluation of the Augustan Age the comparison between Seneca's and Florus' works, along with a new reading of some passages by Florus dedicated to Augustus, shows that the two authors held opposite views on the transition from Republic to Principate. Florus, in fact, seems to ascribe this phenomenon to the wiser and more mature phase almost the acme of the Roman people's life.
\end{abstract}

\section{Premessa}

Delle sole tre testimonianze relative alle Historiae di Seneca Padre in nostro possesso, fino alla riscoperta del papiro P.Herc 1067, due sono da tempo messe in relazione all'opera di Floro, che, nella struttura ${ }^{1}$ e nella divisione per aetates, scelta come criterio narrativo per il racconto della storia di Roma dalle origini ad Augusto, ${ }^{2}$ può aiutare a chiarire alcuni punti relativi ai dati dei testi senecani;

1 L'aspetto certamente più interessante per il confronto con Seneca Padre, sul piano della struttura narrativa, è la divisione netta tra bella externa, narrate tutte nel primo libro fino alle campagne di Gallia di Cesare e la campagna partica di quegli stessi anni, e le "guerre civili", narrate nel secondo libro. Esse iniziano con i moti graccani e finiscono con la battaglia di Azio. La narrazione tuttavia prosegue inglobando parte dell'età Augustea, con un confine cronologico difficile da tracciare perché, fermandosi apparentemente alle campagne di Spagna di Augusto, tratta con un'inversione cronologica anche la disfatta di Varo, di molto successiva rispetto al 27 a.C., anno della consacrazione di Augusto, con cui si chiude l'opera. Tutte le moderne edizioni assumono la partizione dell'opera rappresentata dal solo codice Bamberg, Staatsbibliothek E III 22 (IX sec.), mentre la seconda recensione, a cui appartengono tutti gli altri codici, presenta una divisione in quattro libri. La preferenza per $\mathrm{B}$, che presenta il testo più corretto, ha il pregio di trovare conferma nelle parole dello stesso Floro.

2 Sui problemi relativi alla ripartizione per blocchi di anni e sui problemi testuali dei luoghi che riportano le cifre, cf. in particolare l'introduzione di Jal (1967), quella di Salomone Gaggero 
essa costituisce inoltre una sorta di superamento della periodizzazione di Seneca Padre, in un'epoca diversa, con una cronologia da adeguare, anche in chiave ideologica, alla suddivisione della storia proposta nell'età di Caligola, quando l'opera di Seneca sembra essere circolata. ${ }^{3}$

Il testo del De vita patris, infatti, è stato oggetto di diversi studi, soprattutto perché offre un indizio in merito all'inizio delle Historiae di Seneca: nel ricordare orgogliosamente le doti letterarie del padre, infatti, Seneca figlio aggiunge che l'opera partiva ab initio bellorum civilium, espressione che viene generalmente ricondotta o alla guerra civile tra Cesare e Pompeo ${ }^{4}$ oppure, più recentemente da Canfora, all'età dei Gracchi, ${ }^{5}$ grazie anche alla disposizione degli eventi nell'opera di Floro che colloca l'inizio del secondo libro e delle seditiones interne proprio nel 133 a.C.

Sebbene infatti la Levick, nell'edizione Cornell, adduca fondamentalmente la spiegazione che da Silla alla guerra civile tra Cesare e Pompeo ci sarebbero anni di stasi senza guerre civili e che dunque l'inizio dell'opera senecana dovesse ricadere in un punto più significativo della storia di Roma, ${ }^{6}$ non si possono trascurare gli argomenti di Canfora, che in sintesi afferma: 1. Appiano e Floro, più o meno negli stessi anni, raccontano le guerre civili partendo dai Gracchi e considerano come tappe di esse i tumulti di Saturnino, la guerra sociale, la congiura

(1981), il commento di Facchini Tosi (1997), passim. Una ricapitolazione dei principali problemi connessi alle fonti e ai modelli di Floro per questa concezione biologica della storia in Bessone (2008).

3 Sulla cronologia relativa alla composizione delle Historiae cf. Vottero (1998) 76-77, che ricorda la terza testimonianza relativa all'opera di Seneca Padre che non è utile nel confronto con l'opera di Floro e dunque non sarà oggetto della nostra attenzione perché si riferisce alla morte di Tiberio (riportata da Suet. Tib. 73.2 - Appendix F1), collocazione suffragata da suas. 3.7 in cui l'uso dei tempi sembra confermare che l'imperatore non fosse più in vita mentre Seneca padre scriveva. Per la data della morte di Seneca invece si considera il 41 come terminus ante quem perché il figlio dichiara che la notizia del suo esilio è giunta alla madre già vedova (dial. 12.2.45). Se dunque la morte di Seneca padre si colloca tra il 37 e il 41, il termine del suo racconto dovrebbe ricadere in quegli anni, visto che nella testimonianza del De vita patris il figlio dice del padre che ha scritto fino quasi alla sua morte (paene usque ad mortis suae diem, F 97 Peter). La discussione sui tempi e l'effettiva pubblicazione dell'opera in Levick in FRHist I 507.

4 Ampia la bibliografia sulle diverse periodizzazioni proposte. Cf. Lausberg (1989) 1939 con la bibliografia relativa; contro l'ipotesi dei Gracchi Klotz (1901) 437-438; incerto Sussman (1978) 142143 (cfr. anche 147 n. 18 nel presente volume); possibilista Castiglioni (1928) 458-460, che pur ammettendo quale inizio le guerre civili tra Cesare e Pompeo, pensa ad una visione retrospettiva ad eventi precedenti per l'individuazione dell'initium, la causa prima, per usare la terminologia di Canfora (2015) 138, che si scaglia invece fortemente contro l'ipotesi accolta in FRHist.

5 Canfora (2015) 148 e ss. Cf. anche (2000) 165-168.

6 Levick in FRHist I 506: "the work would lack a crisp starting point". 
di Catilina, la rivoluzione degli schiavi di Spartaco e Crisso, il primo triunvirato, creando quindi un filo continuo dalla seditio Graccana alla battaglia di Azio che di fatto chiude per Floro le guerre civili e apre l'età augustea; 2. l'appartenenza di Floro agli Annaei testimoniata dalla tradizione dell'Epitome rafforza l'ipotesi che Floro possa aver avuto come modello Seneca Padre $^{7}$ e dunque da quest'ultimo avrebbe ereditato l'impostazione della sua storiografia, che individua sostanzialmente un secolo di guerre civili.

Nei manoscritti dell'opera storiografica di Floro l'indicazione Annaeus non è unica, perché la tradizione più autorevole del testo, rappresentata dal codice $\mathrm{B}$ (Bamberg, Staatsbibliothek E III 22) riporta come autore dell'opera storiografica Iulius Florus. ${ }^{8}$ Sebbene sia un caso unico, contro la maggior parte dei testimoni, si tratta del testo assunto da tutte le edizioni moderne, perché presenta la divisione dell'opera in due libri anziché quattro, ritenuta la più verosimile e dunque interessante ai fini della valutazione dell'opera di Seneca Padre proprio perché divide la storia in bella externa e clades domesticae. Tuttavia a causa della massiccia presenza del nome Annaeus negli altri codici e per gli indubbi legami presenti tra i due Seneca, Lucano e Floro, ${ }^{9}$ mi sento di propendere per la soluzione di Canfora che peraltro disgiunge di conseguenza l'opera storica dal resto della produzione ascritta dalla tradizione ad Annio Floro. ${ }^{10}$ Nell'ottica dunque di

7 Ad una dipendenza diretta di Floro, con originalità nel fissare i limiti cronologici pensa già Jal (1967) LXXIV-LXXV e Giacone Deangeli (1969) nell'introduzione al testo di Floro (308); pensa invece ad una fonte comune ai due autori Salomone Gaggero (1981) 47, come già Klotz (1901) che avanza l'ipotesi di una fonte di età augustea.

8 La questione è discussa da Malcovati (1950) 276-279.

9 Molto convincente la posizione di Hahn (1964) 169-206.

10 Sul problema dell'identità di Floro abbiamo cinque attestazioni, giunteci separatamente, di autori con questo nome: a. l'autore dell'epitome compare nei manoscritti come L. Annaeus o Annaeus Florus, mentre in uno dei più importanti manoscritti, B (Bamberg, Staatsbibliothek E III 22), che prevede la divisione in due libri dell'opera adottata da tutte le edizioni più recenti, troviamo Iulius Florus. b. un P. Annius Florus autore del dialogo Vergilius orator an poeta c. un poeta Florus protagonista di uno scambio di poesie con l'imperatore Adriano. d. un funzionario di Adriano citato due volte da Carisio, una volta come Annius Florus e la seconda come Florus e. un poeta di nome Floro, autore di carmi tramandati in un codice dell'Anthologia Latina. Oggi si tende a ritenere che lo stesso autore abbia scritto l'opera storica, il dialogo e la serie di componimenti variamente tramandati, cosicché alcune notizie biografiche contenute nel discorso Vergilius orator an poeta vengono automaticamente considerate utili per meglio comprendere la personalità del Floro storico. Di questa idea già Malcovati (1937) 69-94; 289-307; (1938) 46-64 e soprattutto (1950) 276-279; successivamente Bessone (1996) 123 (idea che ritorna naturalmente anche nei molti altri scritti dell'autore, tra cui (1993b) 80-117, proprio su questo tema); Facchini Tosi (1998) 9; Havas, nella più recente edizione critica (1997) 2, che peraltro sceglie di chiamare l'autore “Annio". Sul problema e i casi presi in esame cf. Baldwin (1988) 134-142. Di recente 
un'appartenenza di Floro alla gens Annaea, un ulteriore confronto tra Seneca Padre e Floro potrà confermare, come vedremo, un elemento affine, che farebbe propendere per la coincidenza dell'incipit dell'opera senecana con l'inizio delle guerre civili del secondo libro di Floro. ${ }^{11}$

Non mi sembra, del resto, che le "prove" a favore di un initium collocabile al 49 siano inoppugnabili: l'incipit del Bellum Civile di Lucano non è prova certa che anche Seneca considerasse quella tra Cesare e Pompeo la guerra civile vera e propria, né le testimonianze relative alle guerre civili disseminate nelle Controversiae e nelle Suasoriae, certo cronologicamente vicine all'esperienza personale dell'autore, ${ }^{12}$ rappresentano una prova dirimente del fatto che il conflitto tra Cesare e Pompeo, peraltro non citato che indirettamente e in modo generico, sia l'incipit dell'opera storica. Certo si può ipotizzare, più cautamente, proprio guardando all'opera di Floro, che l'incipit fossero le seditiones Graccanae cui seguivano tutti gli altri disordini, magari raccontati in modo più sintetico e che poi l'attenzione si attestasse in modo più particolareggiato sulle fasi più vicine alla vita e all'esperienza dell'autore, secondo un uso frequentissimo della storiografia antica.

\section{Il punto di svolta}

Potrebbe aggiungere qualcosa alle precedenti riflessioni la seconda testimonianza ascritta a Seneca Padre, non sempre "ammessa" dagli studiosi, ${ }^{13}$ che secondo me,

sembra incline all'identificazione Perrelli (2017) 131-146. Probabilista, ma con forti perplessità Jal (1967) VII, nella sua introduzione all'edizione.

11 Restano convincenti gli elementi evidenziati da Rossbach (1888) 163-173, ripresi sinteticamente nell'introduzione all'edizione di Floro (1896).

12 Come è noto, le guerre civili fanno in alcuni casi da sfondo ad alcuni testi della raccolta di Seneca Padre. Oltre alla famosa testimonianza biografica, che colloca Seneca Padre lontano da Roma negli anni in cui ancora operava Cicerone (contr. 1 praef. 11), in molti casi la guerra civile è rappresentata attraverso lo scontro tra parenti; la cifra di fondo del dibattimento è l'incertezza sull'applicabilità delle norme consuete in un momento in cui, come per altro avviene sempre nelle declamazioni, la verità non sembra più una sola. Forte è la tentazione di ritrovare anche sullo sfondo delle declamazioni l'idea che la verità sia arretrata irrimediabilmente nella storia come la retorica (cf. 1 praef. 6: nescio qua iniquitate naturae eloquentia se retro tulerit). Sulle declamazioni e le guerre civili, cf., tra gli altri, Danesi Marioni (2003) 151-170, Mazzoli (2006) 4557; Touahri (2010) 55-64.

13 Jal (1967) LXXV, come già Peter (1906) II 68-69, ricorda la teoria, ormai superata, secondo cui Lattanzio, leggendo sul testo di Floro Annaeus, avrebbe ripreso la divisione di Floro attri- 
dialogando con la prima, può offrire ulteriori chiarimenti e generare un confronto utile con Floro.

Nelle Divinae Istitutiones di Lattanzio, infatti, troviamo delineata la concezione biologica della storia, attribuibile a Seneca Padre, forse premessa per le $\mathrm{Hi}$ storiae, in cui la divisione per aetates non sempre è definita da termini cronologici precisi, ma consente forse di capire meglio quando per lo storico è iniziata quella fase di decadenza che ha portato alla rovina di Roma e alla necessità di un cambiamento di regime, inaugurato dall'età augustea: nella divisione in cinque fasi, infatti, il passaggio dalla adulescentia alla iuventus è sancito dalla fine delle guerre puniche e porta alla conquista di tutto il mondo (Appendix - F2). ${ }^{14}$

Come si può notare, l'età delle vittorie, la iuventus, sembra coincidere con un periodo che va dalla presa di Cartagine alla conquista dell'orbe, ma questa determinazione è generica e offre soprattutto la possibilità di sviluppare un tema etico importante per Seneca come anche per Floro: l'idea cioè che i successi militari, evidentemente rappresentati in modo iperbolico dall'idea che fosse finita la bellorum materia, (corrispondente alla nimia felicitas raggiunta da Roma, per Floro, a seguito delle grandi conquiste) abbiano prodotto all'interno un fenomeno degenerativo segnato dagli scontri civili. ${ }^{15}$ Anche la iuventus di Floro, ${ }^{16}$ pur iniziando con le guerre puniche, si configura come una età a due facce, divisa addirittura dall'autore internamente in cento anni aurei, quelli delle grandi conquiste, e cento anni ferrei, in cui nonostante le conquiste continuassero, i Romani erano afflitti dalle guerre civili: a epit. 1.19, infatti, egli individua nella presa di

buendola ad un imprecisato Seneca. Troppe tuttavia, come vedremo, le differenze nelle due periodizzazioni, anche se dubbi restano sulla formalizzazione del testo, per alcuni più vicino alla prosa di Lattanzio, come in Hartke (1951) 393, e per altri caratterizzato dalla numerosa presenza del quasi, su cui cf. Bessone (2008) 35. Una ricognizione delle principali posizioni, con ampia bibliografia in Griffin (1972) 19. Molto sommaria la discussione e la bibliografia offerta da Levick in FRHist I 597. Resta prevalente oggi la linea che ascrive la testimonianza a Seneca Padre: cf. Castiglioni (1928); Lausberg (1970) 3, e (1989) 1957; Bocciolini Palagi (1978) 220, Vottero (1998) 75-81 (che infatti non inserisce il testo nei frammenti di Seneca figlio) e naturalmente Canfora (2000) 165 e (2015) 158.

14 Lact. inst. 7.15.14.

15 Cf. anche la lettura di Sussman (1978) 149: "The date for the beginning of the decline is the destruction of Carthage and the emergence of the Gracchi"; cf. anche lo stesso Sussman supra 175-177.

16 Cf. Flor. epit. 1.18: domita subactaque Italia populus Romanus prope quingentensimum annum agens cum bona fide adolevisset, si quod est robur, si qua iuventas, tum ille vere robustus et iuvenis et par orbi terrarum esse coepit. Ita - mirum et incredibile dictu - qui prope quingentis annis domi luctatus est - adeo difficile fuerat dare Italiae caput - his ducentis annis qui secuntur Africam, Europam, Asiam, totum denique orbem terrarum bellis victorisque peragravit. 
Numanzia il termine cronologico di una fase della iuventus tutta positiva, nei cento anni dedicati alla conquista di Africa, Macedonia, Sicilia e Spagna (264133), mentre i successivi, precisa:

centum sequentes ferreos plane et cruentos et si quid inmanius; quippe qui Iugurthinis, Cimbricis, Mithridaticis, Parthicis, piraticis bellis, Gallicis atque Germanicis, quibus caelum ipsum gloria ascendit Gracchanas Drusianasque caedes, ad hoc servilia bella miscuerint et, ne quid turpitudini desit, gladiatoria. Denique in se ipse conversus Marianis atque Sullanis, novissime Pompei et Caesaris manibus, quasi per rabiem et furorem - nefas! - semet ipse laceravit. $(1.34 .19)^{17}$

Si pone dunque un discrimine tra le conquiste che ruota comunque intorno al 133, data della battaglia di Numanzia e dell'eredità di Attalo III, che costituiscono senza dubbio il culmine di successo all'estero dell'impero romano conquistatore, ma anche, per Floro, quel raggiungimento di un'eccessiva prosperità che ha generato le guerre civili; a epit. 1.47, infatti, Floro argomenta:

posteri centum, quos a Carthaginis, Corinthi Numantiaeque excidiis et Attali regis Asiatica hereditate deduximus in Caesarem et Pompeium secutumque hos, de quo dicemus, Augustum, ut claritate rerum bellicarum magnifici, ita domesticis cladibus miseri et erubescendi. (epit. 1.47.3)

Il momento di crisi è individuato e commentato nella volontà di conquista:

ac nescio an satius fuerit populo Romano Sicilia et Africa contento fuisse, aut his etiam ipsis carere dominanti in Italia sua, quam eo magnitudinis crescere, ut viribus suis conficeretur. Quae enim res alia civiles furores peperit quam nimia felicitas? Syria prima nos victa corrupit, mox Asiatica Pergameni regis hereditas. (epit. 1.47.6)

Il testo di Floro è indubitabilmente vicino alla considerazione di Seneca Padre: tutti e due colgono la volontà di conquista del mondo come inizio della iuventus (manus suas in totum orbem terra marique porrexit, in Seneca e orbe toto arma circumtulit, in Flor. epit.1.47.3), ma successivamente si evidenzia la conseguenza della scelta: donec regibus cunctis et nationibus imperio subiugatis cum iam bellorum materia deficeret, viribus suis male uteretur, quibus se ipsa confecit, afferma Seneca, sottolineando così che, terminate le guerre esterne, Roma ha distrutto se stessa; su questa idea concorda anche Floro: ac nescio an satius fuerit populo Romano Sicilia et Africa contento fuisse, aut his etiam ipsis carere domi-

17 Il testo seguito è quello di Malcovati $\left(1972^{2}\right)$. 
nanti in Italia sua, quam eo magnitudinis crescere, ut virtutibus suis conficeretur $^{18}$ che riprende la stessa espressione per indicare l'inizio della crisi; entrambi infine stigmatizzano i conflitti interni che si sono generati: Et haec fuit prima eius senectus, cum bellis lacerata civilibus atque intestino malo pressa rursus ad regimen singularis imperii recidit quasi ad alteram infantiam revoluta, afferma Seneca seguito da Floro, che approfondisce ed elenca tutte le seditiones partendo dai Gracchi e anticipando la definitiva soluzione con l'avvento di Augusto (epit. 1.47.8-14). Se dunque Floro segnala un cambiamento importante nelle conquiste esterne databile al 133, per valorizzare il nesso di passaggio con la rivolta dei Gracchi, ${ }^{19}$ anch'essa di quell'anno, e per stabilire un punto di svolta nella storia di Roma, continuando a raccontare (relegate nel primo libro) le successive conquiste, potremmo cogliere anche nella generica riflessione del Seneca riportata da Lattanzio una condanna etica affine a quella di Floro, di cui potrebbe essere stato il modello, che potrebbe riflettere dunque il momento di inizio della decadenza proprio nel 133. Questo dato potrebbe accrescere la credibilità della proposta, sostenuta da Canfora, di individuare nella seditio Graccana del 133 l'inizio della prima senectus, in cui peraltro l'aggettivo mi sembra rafforzare l'idea di $a b$ initio bellorum civilium espressa dalle parole del figlio. D’altronde non mi sembra possibile proporre una periodizzazione senecana che consideri una effettiva conclusione delle conquiste esterne, visto che esse non ebbero mai fine praticamente, se non riconoscendo un momento storico che attivi una riflessione etica e che dunque conferisca la giusta dignità all'incipit narrativo scelto per la propria opera storica.

\subsection{Il passaggio all'età augustea}

Nella definizione delle aetates lo scarto forse maggiore tra i due scrittori riguarda proprio il passaggio al principato, che, oltre a tradire un atteggiamento ideologico assolutamente opposto, consente a Floro una periodizzazione più congruente con l'angolazione da cui un uomo dell'età degli Antonini può effettivamente valutare l'evoluzione della storia di Roma. ${ }^{20}$

18 Il concetto è già in Liv. praef. 4: res est praeterea et immensi operis, ut quae supra septingentesimum annum repetatur et quae ab exiguis profecta initiis eo creverit ut iam magnitudine laboret sua; et legentium plerisque haud dubito quin primae origines proximaque originibus minus praebitura voluptatis sint, festinantibus ad haec nova quibus iam pridem praevalentis populi vires se ipsae conficiunt.

19 Cf. Havas (1994) 27.

20 Garzetti (1964) 136-156. 
Nel passo riportato da Lattanzio, infatti, quella prima senectus iniziata in un tempo lontano e caratterizzata dall'autodistruzione delle guerre civili, prosegue rursus ad regimen singularis imperii, costringendo il popolo romano a riacquistare l'ordinamento della sua infantia (quasi ad alteram infantia revoluta). La regressione, con conseguente perdita della libertà, contiene un evidente giudizio polemico, come si può cogliere dall'espressione finale: amissa enim libertate, quam Bruto duce et auctore defenderat, ita consenuit, tamquam sustentare se ipsa non valeret, nisi adminiculo regentium niteretur. La necessità di un adminiculum regentium, senza il quale la senectus non è in grado di sostenersi, genera il rimpianto per la perdita di quella libertà conquistata proprio in un movimento inverso a quello che ha portato al principato, quando in un lontano passato si era passati dai reges alla res publica: mi sembra infatti molto più naturale pensare che l'allusione in questo luogo sia a Lucio Giunio Bruto che a Marco Bruto, ${ }^{21}$ perché l'autore crea un parallelo tra il passato e il futuro, posti in direzioni opposte. Nella prima infantia, infatti, si assiste ad una crescita, nella seconda ad un'involuzione verso la condizione di schiavitù faticosamente combattuta con la cacciata di Tarquinio. L'idea della amissa libertas e l'obbligo nei confronti dei "reggitori" naturalmente si associa all'idea di una veritas perduta (come dice Seneca nel de vita patris: unde primum veritas retro abiit) quando il motore delle azioni del popolo romano non è stato più unitario, ma legato all'interesse personale di alcuni. Naturalmente Seneca, che "attraversa” l'età augustea, doveva avere ben chiaro in che termini la libertas e la veritas al suo tempo fossero fortemente limitate dietro la facciata del ritorno alla res publica. È lui stesso a darcene testimonianza a proposito di Augusto nelle Controversie: se infatti Seneca ricorda la libertà di parola dell'epoca, tanto da mostrare un princeps indulgente quando un po' a sproposito emergono in un discorso le origini oscure di Agrippa (contr. 2.4.12-13), ricorda anche il giro di vite del princeps sulla produzione letteraria, quando proprio

21 Pensa a Marco Bruto Castiglioni (1928) 460, ma come sottolineano Griffin (1972) 19 e Levick in FRHist I 597, sembra più giusto propendere per Lucio. Mi sento di aggiungere che a torto è stato invocato proprio Floro a sostegno dell'ipotesi di Marco Bruto, nei riguardi del quale per esempio Bessone (2004-2005) 305-324, ritiene che Floro abbia una particolare propensione. In realtà nel racconto di Floro i cesaricidi sembravano aver restaurato la libertà perduta con l'omicidio di Cesare, ma, aggiunge l'autore, libertatem quam maxime restitutam voluerunt, illo ipso parricidio perdiderunt (epit. 2.17.1). L'azione di Ottaviano, inoltre, è tutta mossa a vendicare la morte del padre e la sconfitta dei cesaricidi è motivata dall'agire della Fortuna che favorisce la sua ascesa (stabat tamen pro partibus invicta fortuna et ultoris et qui vindicabatur ut exitus proelii docuit, epit. 2.17.10) e che nella circostanza ha la meglio sulla virtù (sed quanto efficacior est fortuna quam virtus!, epit. 2.17.10) nel percorso di affermazione di Ottaviano. Su virtus e fortuna in Floro cf. Nordh (1952) 111-128 e Cupaiuolo (1984) 34-35. 
un'opera storiografica, come quella di Labieno, ben più incisiva perché scritta per orientare la memoria, il pensiero, e dunque il consenso, ${ }^{22} \mathrm{fu}$ data alle fiamme e il suo autore si seppellì vivo nella tomba di famiglia. ${ }^{23}$

Mentre dunque la periodizzazione di Seneca sembra includere Augusto nella senectus, di cui il principato è logica conseguenza, di tutt'altro segno appare la divisione per aetates di Floro, ${ }^{24}$ che a proposito della iuventus e dei tempi successivi scrive:

deinceps ad Caesarem Augustum centum et quinquaginta anni, quibus totum orbem pacavit. Hic iam ipsa iuventas imperii et quaedam quasi robusta maturitas. A Caesare Augusto in saeculum nostrum haud multo minus anni ducenti, quibus inertia Caesarum quasi consenuit atque decoxit, nisi quod sub Traiano principe movit lacertos et praeter spem omnium senectus imperii quasi reddita iuventute revirescit. (epit. 1 praef. $7-8$ )

Anche questo passaggio presenta diverse difficoltà: la iuventus, altrove più volte conteggiata in 100 anni aurei e 100 ferrei, arriva ad includere anche Cesare Augusto, a cui è del resto dedicata larga parte del secondo libro. ${ }^{25}$ Floro stabilisce però con chiarezza che la battaglia di Azio chiude l'età delle guerre civili, creando un nesso di passaggio importante per gli eventi dell'età augustea: sebbene non si debba intendere una vera e propria periodizzazione interna a questa età, l'espressione hic iam ipsa iuventus imperii et quaedam quasi robusta maturitas consente una riflessione ulteriore che definisce in modo perspicuo gli anni di Augusto. Per spiegare il passaggio dalla repubblica al principato, infatti, Floro parte da una considerazione importante: dopo la morte di Cesare, Roma sarebbe potuta tornare all'antica libertà, se questi non avesse lasciato degli heredes smaniosi di proseguire il cammino ormai iniziato. Dopo questa breve concessione nostalgica, che rivela peraltro una lucida valutazione politica e sembra tradire l'avversione per il principio ereditario del potere, "il suo giudizio su Ottaviano risulta ampiamente positivo, come d'altronde lo sarà sul princeps". ${ }^{26}$ Sebbene non si possa esaurire in queste pagine l'analisi del racconto del principato augusteo di Floro, ${ }^{27}$ bisogna ricordare che per l'autore, dopo i disordini seguiti alla morte di Cesare,

22 De Vivo (1998) 9-27.

23 Borgo (2012) 33-53. Sull'immagine di Augusto nell'opera di Seneca Padre, cf. Cogitore (2016) 195-208.

24 Sullo schema biologico di Floro Hahn (1965) 21-38 e in relazione ad altre periodizzazioni nella storiografia Paschoud (2002) 323-321.

25 Cf. Bessone (2008) 21.

26 Bessone (2004-2005) 307.

27 Manca uno studio complessivo sul tema, per alcuni aspetti trattato da Bessone (1993a) 93100; (2002-2003) 21-36; (2004-2005) 305-324; Jal (1965) 358-383; Havas (1994) 21-29. 
non vi era altro esito che il passaggio alla servitù, ${ }^{28}$ fortunatamente nelle mani di Ottaviano, ritratto come uomo di grande saggezza, che inizia una nuova fase di stabilità e di pace:

gratulandum tamen ut in tanta perturbatione est, quod potissimum ad Octavium Caesarem Augustum summa rerum redit, qui sapientia sua atque sollertia perculsum undique ac perturbatum ordinavit imperii corpus, quod haud dubie numquam coire et consentire potuisset, nisi unius praesidis nutu quasi anima et mente regeretur. (epit. 2.14.5)

Il richiamo alla metafora del corpo, già premessa necessaria a quella delle aetates del popolo Romano, si rafforza con l'idea che tale corpus sia nelle mani di uno solo, che lo gestisce con il cenno del capo; è un passaggio importante, l'inizio di una fase nuova, come sembra confermato dall'ultimo capitolo dell'opera, interamente dedicato alla consecratio Augusti:

sic ubique cuncta atque continua totius generis humani aut pax fuit aut pactio, aususque tandem Caesar Augustus septingentesimo ab urbe condita anno Ianum geminum cludere, bis ante se clusum sub Numa rege et victa primum Carthagine.

Hinc conversus ad pacem pronum in omnia mala et in luxuriam fluens saeculum gravibus severisque legibus multis coercuit, ob haec tot facta ingentia dictator perpetuus et pater patriae. ${ }^{29}$ (epit. 2.34.64-65).

In quest'ottica la riflessione del primo passo e le considerazioni della conclusione dell'opera potrebbero ridare valore e spiegare meglio l'espressione et quaedam quasi robusta maturitas della praefatio, generalmente schiacciata sulla iuventus, così da non costituire un ulteriore frazionamento delle aetates. Facchini Tosi, infatti, argomenta: "Per Floro maturitas è sinonimo di iuventas...l'irruenza dell'adolescenza lascia ora il posto alla pacatezza della maturitas, di un rigoglio di forze". ${ }^{30}$ Pur concordando sul fatto che iuventas e maturitas si trovino entrambe

28 Flor. epit. 2.14.4: nam aliter salvus esse non potuit, nisi confugisset ad servitutem. Il luogo è in realtà compromesso da una lacuna immediatamente precedente a nam, per cui il soggetto, per la maggior parte degli editori un sottinteso populus Romanus, non è del tutto certo. Segnaliamo per completezza le osservazioni e le proposte di integrazione di Bessone (2004-2005) 318-320.

29 Sul concetto di "dittatore perpetuo" da intendersi come errore di Floro mutuato forse da una sua fonte, già in età imperiale avvertito come corrispondente all'istituzione del principato, cf. Bessone (1978) 421-431.

30 Facchini Tosi (1998) 105. A favore di un'età unica "robusta" anche Flor. epit. 1.18.1(1): domita subactaque Italia populus Romanus prope quingentensimum annum agens cum bona fide adolevisset, si quod est robur, si qua iuventas, tum ille vere robustus et iuvenis et par orbi terrarum esse coepit. La maturitas compare invece come età a sé stante nella più tarda periodizzazione proposta da Tertulliano, secondo Tibiletti (1959) 339-342 influenzato dal modello di Seneca e Floro. 
nella terza età di Floro, in cui per ammissione di tutti gli studiosi va inteso anche il principato augusteo, ${ }^{31}$ potremmo cogliere una raffinata "evoluzione interna", volutamente espressa dall'autore nel passaggio alla maturitas, intesa dagli antichi come una fase particolare della vita. Il concetto di maturitas, infatti, è tecnicamente legato all'evoluzione delle piante, come è facile immaginare, ${ }^{32}$ ma sorprendentemente poco attestato per indicare un momento preciso della vita umana. Si tratta di una parola usata in tale accezione da Cicerone, in passi in cui questa fase è definita in opposizione ad altre: nella pro Caelio, per esempio, è l'età della saggezza, contrapposta all'adulescentia:

sed ego non loquor de sapientia, quae non cadit in hanc aetatem; de impetu animi loquor, de cupiditate vincendi, de ardore mentis ad gloriam; quae studia in his iam aetatibus nostris contractiora esse debent, in adulescentia vero tamquam in herbis significant, quae virtutis maturitas et quantae fruges industriae sint futurae. (Cael. 76.10)

Cicerone sottolinea l'evoluzione dall'irruenza della gioventù alla pacatezza della maturità, ${ }^{33}$ un aspetto che nella periodizzazione di Floro si riferisce meglio all'età augustea della pax che agli anni irruenti delle guerre di conquista e a quelli burrascosi delle guerre civili (iuventus di Floro). Così la maturitas diviene nel Cato

31 Uno dei problemi più spinosi della periodizzazione di Floro è stabilire il confine "storico" tra la fine della iuventus e l'inizio della senectus; se infatti l'età augustea va inserita nella terza età, non si comprende bene la determinazione generica della praefatio, che fa giungere ad Augusto la giovinezza (epit. 1 praef. 7: deinceps ad Caesarem Augustum centum et quinquaginta anni, quibus totum orbem pacavit. Hic iam ipsa iuventus imperii et quaedam quasi robusta maturitas), ma fa anche ripartire la senectus dall'età di Augusto (epit. 1 praef. 8: a Caesare Augusto in saeculum nostrum haud multo minus anni ducenti, quibus inertia Caesarum quasi consenuit atque decoxit, nisi quod sub Traiano principe movit lacertos et praeter spem omnium senectus imperii quasi reddita iuventute revirescit). Se consideriamo i limiti effettivi della narrazione, arriviamo all'assunzione del titolo di Augusto nel 27 a. C., ma ci sono riferimenti alle leggi emanate in anni successivi. Si tratta di un dato importante da calcolare, perché implica anche la determinazione dell'epoca di Floro (a Caesare Augusto in saeculum nostrum). Gli studiosi di Floro hanno valutato una serie di ipotesi, coincidenti con le date importanti connesse al principato: la data di nascita di Ottaviano, il 43, età del primo consolato, gli anni 30-27, in cui assunse il potere assoluto, e il 14 d.C., data della morte. Jal (1967) XCIV-CII, ridiscutendo queste opzioni, si dice convinto del 43 come data di inizio del regime augusteo (percepita anche da altri importanti storici) e la sceglie per calcolare l'età di composizione dell'Epitome. Resta la perplessità relativa alla scelta di completare la narrazione al 27, che sembrerebbe concretamente il discrimine storico avvertito da Floro come più importante per indicare il potere assoluto di Augusto.

32 Cf. ThlL VIII col. 493 1. 16 - col. 495 1. 31.

33 Cf. la stessa opposizione in Cic. orat. 30.107: sunt enim omnia sic ut adulescentis non tam re et maturitate quam spe et exspectatione laudati. Ab hac etiam indole iam illa matura. 
Maior una prerogativa quasi della senilità, con cui viene indissolubilmente connessa e appare dunque lontanissima da quella iuventas cui viene, nelle considerazioni su Floro, impropriamente sovrapposta:

cursus est certus aetatis et una via naturae, eaque simplex, suaque cuique parti aetatis tempestivitas est data, ut et infirmitas puerorum, et ferocitas iuvenum et gravitas iam constantis aetatis et senectutis maturitas naturale quiddam habeat, quod suo tempore percipi debeat. (Cato 33)

Ma il passo forse più interessante con cui confrontare le aetates di Floro è Cic. Brut. 2.8:

ita nobismet ipsis accidit ut, quamquam essent multo magis alia lugenda, tamen hoc doleremus quod, quo tempore aetas nostra perfuncta rebus amplissimis tamquam in portum confugere deberet non inertiae neque desidiae, sed oti moderati atque honesti, cumque ipsa oratio iam nostra canesceret haberetque suam quandam maturitatem et quasi senectutem, tum arma sunt ea sumpta, quibus illi ipsi, qui didicerant eis uti gloriose, quem ad modum salutariter uterentur non reperiebant.

Cicerone, parlando della sua esperienza personale, riferendo il frazionamento per aetates alla sua propria oratoria, dice che nella maturità, quasi senectus, l'attività non dovrebbe tramutarsi in inertia, ma in un ozio moderato ed onesto. In questo caso la presenza di maturitas e senectus sembra indicare due fasi contigue in un momento di trapasso dall'una all'altra come nel caso della iuventas et quaedam quasi maturitas di Floro, in cui non c'è soluzione di continuità certo, ma nemmeno una coincidenza precisa, per la presenza intenzionale di et con valore disgiuntivo e, soprattutto, del quaedam, che compare in entrambe i passi e dissolve i confini di una sovrapposizione certa, ma anzi rafforza in Floro il momento di transizione delle due fasi. Va segnalato che l'ambito "metaforico" da cui parte il concetto di maturitas può meglio spiegare il senso del suo uso per la vita umana: Gellio (10.11.2-3), infatti, richiamandosi a Nigidio e proprio alle fasi di maturazione del frutto, dà la definizione più compiuta del termine:

P. Nigidius, homo in omnium bonarum artium disciplinis egregius: 'mature' inquit 'est quod neque citius est neque serius, sed medium quiddam et temperatum est'. Bene atque proprie Nigidius. Nam et in frugibus et in pomis 'matura' dicuntur, quae neque cruda et inmitia sunt neque caduca et decocta, sed tempore suo adulta maturataque.

Gellio dice infatti che la maturitas si colloca tra la fase acerba e aspra e quella ormai cadente e appassita (decocta) del frutto. Floro assume il lessico della botanica e sovrappone alla metafora delle aetates dell'uomo, per spiegare le fasi della 
storia di Roma, la crescita di un frutto, ${ }^{34}$ così da dare concretamente il senso dell'evoluzione alle diverse fasi della "vita" del popolo Romano: se infatti la iuventus viene determinata "nel fiore degli anni" (ut quasi ad quandam iuventae frugem pervenerit, Flor. epit. 1 praef. 5), questa stessa età trascorre fino ad una robusta maturitas, coincidente con gli anni del potere di Augusto e si configura come momento di passaggio limitrofo alla vecchiaia, in cui il popolo decoxit, letteralmente "appassì" (cf. Gell. 10.11.3: neque caduca et decocta) nell'inertia dell'età giulio-claudia, per "rinverdire” (miracolosamente: praeter spem omnium) con l'avvento di Traiano.

Se consideriamo il particolare approccio di Floro alla periodizzazione storica, spesso più orientato a dare una veste retorica alla sua opera che non alla precisione cronologica, con la "fioritura" di immagini che colpiscano l'immaginario del lettore, la maturitas rappresenta per Floro il "ponte" tra l'ultima fase della iuventus e la successiva senectus di cui, secondo le testimonianze del suo uso, costituisce il momento immediatamente precedente e con cui talvolta quasi si identifica. ${ }^{35}$ Essa potrebbe dunque rappresentare la fase della storia romana segnata dall'avanzata di Ottaviano, risolutore delle guerre civili e fondatore dell'età dei Cesari, anche se non è però conteggiato tra essi, perché collocato in una "fase precedente”, cioè la maturitas della praefatio. Il primo principato viene dunque a rappresentare un momento indefinibile tra due "età", come appaiono del resto nel racconto di Floro gli anni del potere di Ottaviano/Augusto, i cui confini anche cronologici restano difficilmente delineabili. Certo la prospettiva dell'età degli Antonini, in cui al modello augusteo già Adriano sembra richiamarsi fortemente, può forse spiegare lo strano "salto generazionale" compiuto dal popolo Romano: dopo la senectus ricomincia il suo ciclo ${ }^{36}$ con l'età "rigogliosa" di Traiano e nel

34 Hamblenne (1985) 623-626.

35 Cf. per esempio, Cic. Cato 71.13, dove alla maturazione dei frutti corrisponde la senilità dell'uomo: et quasi poma ex arboribus, cruda si sunt, vix evelluntur, si matura et cocta, decidunt, sic vitam adulescentibus vis aufert, senibus maturitas.

36 La divisione in quattro fasi, infatti, scelta da Floro per le sue aetates affonda in un'antica tradizione, probabilmente di matrice pitagorica, che vede la corrispondenza tra le età dell'uomo e le stagioni della natura, secondo l'immagine offerta da Ovidio (met. 15.199-213): secondo il poeta, infatti, alla primavera corrisponde tener et lactens puerique simillimus aevo, all'estate un valens iuvenis, robusto e ardente, all'autunno la maturitas (ben diversa dalla gioventù!) così caratterizzata: posito fervore iuventae / maturus mitisque, inter iuvenemque senemque / temperie medius, sparsus quoque tempora canis; infine segue l'inverno, corrispondente alla nostra vecchiaia: inde senilis hiems tremulo venit horrida passu, / aut spoliata suos, aut, quos habet, alba capillos. Sulla divisione in quattro età e la visione ciclica dunque del tempo, Ruch (1972) 827841. 
rinvigorire delle forze sembra superare quella revoluta infantia che nella riflessione di Seneca Padre portava con sé traccia dell'involuzione del principato. Con tale passaggio e il sensibile cambio di segno di questa valutazione di Floro, Roma può dunque ripartire così da quella iuventus et quasi robusta maturitas culminante al tempo di Augusto, nell'ottica tutta ottimistica della felicitas temporum. 